\title{
Therapeutic potential of sepantronium bromide YM155 in gemcitabine-resistant human urothelial carcinoma cells
}

\author{
YEN TA HUANG ${ }^{1-3}$, CHUAN CHU CHENG ${ }^{4}$, TZU CHUN LIN ${ }^{4}$, TED H. CHIU ${ }^{1}$ and PEI CHUN LAI ${ }^{2,5}$ \\ Departments of ${ }^{1}$ Pharmacology, and ${ }^{2}$ Medicine, Tzu Chi University, Hualien; \\ ${ }^{3}$ Surgical Intensive Care Unit; Departments of ${ }^{4}$ Medical Research and ${ }^{5}$ Pediatrics, \\ Buddhist Tzu Chi General Hospital, Hualien, Taiwan, R.O.C.
}

Received October 15, 2013; Accepted November 4, 2013

DOI: $10.3892 /$ or.2013.2882

\begin{abstract}
Survivin is overexpressed in transitional cell carcinoma (TCC), the most common type of bladder cancer. Previous reports demonstrated that knockdown of survivin by siRNA induced apoptosis of TCC cells. The present study evaluated the therapeutic effects of sepantronium bromide (YM155), a novel small molecule survivin inhibitor under clinical trials, on TCC cells in vitro. BFTC905, a grade III TCC cell line derived from a patient of blackfoot disease in Taiwan, was the most gemcitabine-resistant cell line when compared to BFTC909, TSGH8301 and T24 in cytotoxicity assay, resulting from upregulation of securin and bcl-2 after gemcitabine treatment. YM155 caused potent concentration-dependent cytotoxicity in 4 TCC cell lines $\left(\mathrm{IC}_{50} \mathrm{~s} \leq 20 \mathrm{nM}\right)$, but exhibited no cytotoxicity in survivin-null primary human urothelial cells. For BFTC905 cells, addition of gemcitabine and/or cisplatin, the standard TCC chemotherapy regimen, to YM155 did not exert additive cytotoxic effects. Molecular analyses indicated that YM155 inhibited the proliferation of BFTC905 cells by increasing p27 ${ }^{\mathrm{kip} 1}$, suppressing $\mathrm{Ki}-67$, and inducing quiescence. In addition, YM155 elicited apoptosis manifested with DNA fragmentation through suppressing the expression of survivin, securin and bcl-2. Furthermore, YM155 induced autophagy in BFTC905 cells as autophagic inhibitor, 3-methyladenine, attenuated YM155-induced LC3B-II levels and reversed the cytotoxicity of YM155. mTOR inhibitors sirolimus and everolimus did not increase YM155-induced expression of LC3B-II nor augment YM155-induced cytotoxicity. These results indicate that YM155 exerts its lethal effect on BFTC905 cells via apoptotic and autophagic death
\end{abstract}

Correspondence to: Dr Pei Chun Lai, Department of Pediatrics, Buddhist Tzu Chi General Hospital, 707 Chung Yang Road, Section 3, Hualien 970, Taiwan, R.O.C.

E-mail: chunta.lab@gmail.com

Key words: gemcitabine, YM155, survivin, bladder cancer, transitional cell carcinoma pathways and suggest that YM155 may be a potential drug for the therapy of gemcitabine-resistant bladder cancer.

\section{Introduction}

Urinary bladder cancer, pathologically presented mainly as transitional cell carcinoma (TCC) (1), is one of the most common malignancies in the world (2). Once diagnosed as a muscle-invasive type, the 5-year survival and freedom from relapse rates under conservative multimodality therapies were 54 and 42\%, respectively (3). Chemotherapy with standard regimen methotrexate, vinblastine, adriamycin and cisplatin (MVAC) or more tolerable regimen GC (gemcitabine and cisplatin) for advanced or metastatic bladder cancer has demonstrated poor response (4). Currently, due to cisplatin-induced nephrotoxicity, gemcitabine is the chief strategy for bladder cancer therapy (5). However, fair prognosis was noted in several trials of gemcitabine plus other anti-neoplastic drugs $(6,7)$. Gemcitabine resistance in TCC cells has been noted (8).

Survivin, a member of the inhibitor of apoptosis protein family, has been shown to be a diagnostic biomarker and prognostic parameter of bladder cancer (9). Sepantronium bromide, a small molecule also known as YM155, directly binds to the transcription factor ILF3/NF110 or disrupts ILF3/p54 ${ }^{\text {nrb }}$ complex to inhibit the activity of survivin gene promoter $(10,11)$. YM155 has been demonstrated to inhibit the progression of certain neoplasms, such as prostate $(10,12)$ and lung cancer $(13,14)$. In the present study, we demonstrated that gemcitabine-resistant human TCC BFTC905 cells were sensitive to YM155 at very low concentrations, and the antineoplastic effects were more potent than cisplatin and/or gemcitabine. Notably, YM155 did not elicit cytotoxic effects on primary human urothelial cells. YM155 may be a suitable candidate for TCC that is difficult to treat in the future.

\section{Materials and methods}

Cell lines and cell culture. BFTC905 (15), BFTC909 (15), T24 and TSGH8301 TCC cell lines were maintained as previously described (16,17). Primary human urothelial cells (HUCs) were also used and cultured in the special medium as suggested (\#4312) (both from ScienCell Research Laboratories, Carlsbad, CA, USA). 
MTT cytotoxicity assay. Procedures were followed as previously described $(16,17)$. YM155 (Selleck, Houston, TX, USA), cisplatin (Sigma, St. Louis, MO, USA), sirolimus and everolimus (both from LC Laboratories, Woburn, MA, USA) were dissolved in DMSO (J.T. Baker, Phillipsburg, NJ, USA). Gemcitabine and 3-methyladenine (3-MA) (both from Sigma) were dissolved in distilled water.

Proliferation assay. For proliferation detection, the growth of $1 \times 10^{5}$ TCC cells or HUCs was determined in a $10 \mathrm{~cm}$ dish with complete medium plus YM155 or DMSO at $0 \mathrm{~h}$. After $24 \mathrm{~h}$, the medium was removed and the cells were treated as before. Cell counts were calculated by a hemocytometer $48 \mathrm{~h}$ later.

Cell cycle analysis. BFTC 905 cells were plated at a density of $2 \times 10^{5}$ cells $/ 60-\mathrm{mm}$ petri dish. After treatment, the surfactant and the dead cells were removed. Only the cell cycle changes in the viable cells were analyzed. Procedures using flow cytometry (BD Biosciences, Bedford, MA, USA) as previously described were followed (18).

Immunofluorescent staining. Hoechst 33342 (Enzo Life Sciences, Farmingdale, NY, USA) staining was used for nuclei labeling. Expression of phospho-histone $\mathrm{H}_{2} \mathrm{AX}$ (\#9719) and cleaved poly(ADP-ribose) polymerase [PARP (\#9418); both from Cell Signaling Technology, Inc., Danvers, MA, USA] were measured by the fluorescent intensity in flow cytometry. Cyto-ID ${ }^{\circledR}$ Autophagy Detection kit (Enzo Life Sciences) was used for staining of autophagic vesicles. For semi-quantitation of condensed nuclei, cells were chosen randomly from 10 fields and photographed by Olympus fluorescence microscope BX43 (x400) plus Panasonic DMC-G1 digital camera.

Western blot analysis. Conventional procedures were followed as previously described (16). Primary antibodies were purchased from Cell Signaling Technology, Inc., with anti-survivin (\#2808), anti-LC3B (\#2775), cell cycle regulation (\#9932) and cell cycle/checkpoint antibody sampler kits (\#9917), Abcam (Cambridge, MA, USA) with anti-securin (ab3305), GeneTex, (Irvine, CA, USA) with anti-bcl2 (GTX127958), anti-TCTP (GTX63597), anticyclin A1 (GXT103042), anti-cyclin B1 (GTX100911), anti-cyclin D1 (GTX112874), cyclin E1 (GTX103045) Santa Cruz Biotechnology, Inc. (Dallas, TX, USA) with antiKi67 (sc-15402), $\alpha$-tubulin (sc-8305) and actin (sc-1616). Expression of $\alpha$-tubulin or actin was used as the internal standard.

DNA fragment assay. The Cell Death Detection ELISA ${ }^{\text {plus }}$ (Roche, Mannheim, Germany) assay kit was used to differentiate apoptotic or necrotic condition of $2 \times 10^{4}$ BFTC 905 cells after YM155 treatment. Procedures were followed as previously described (19).

Statistical analysis. Data are presented as means \pm SEM (standard error of mean) and analyzed by Student's t-test or two-way ANOVA based on individual data. In all cases, $p<0.05$ was considered to indicate a statistically significant difference and is labeled by ${ }^{*}$ in the figures. ${ }^{* *} \mathrm{p}<0.01$ and ${ }^{* * *} \mathrm{p}<0.005$.

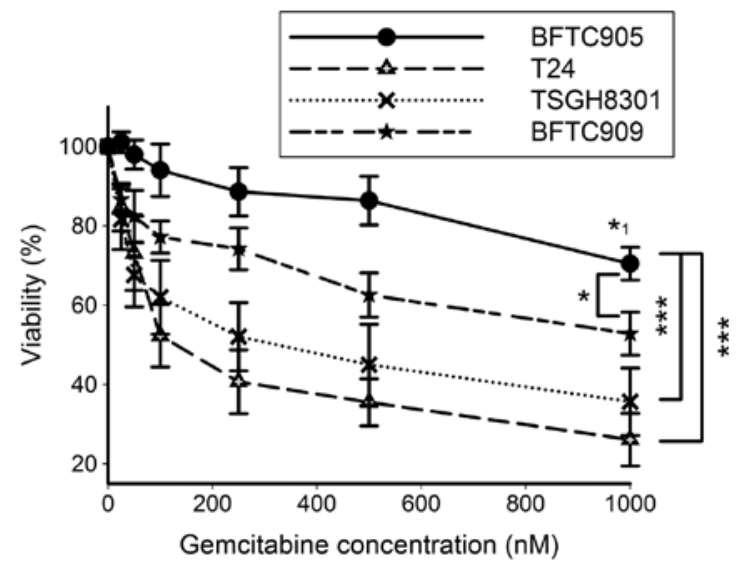

Figure 1. Cytotoxicity of gemcitabine in TCC cells. The viability of BFTC905, T24, TSGH8301 and BFTC909 cells was measured by MTT assay $48 \mathrm{~h}$ after treatment with gemcitabine. The viability ratio was the ratio of the OD value from the drug-treated sample divided by that of the control sample. ${ }^{* 1} \mathrm{p}<0.05$ between the difference in viability of BFTC905 cells after 1,000 nM gemcitabine and control. ${ }^{* * *} \mathrm{p}<0.005$. TCC, transitional cell carcinoma.

\section{Results}

Cytotoxicity of gemcitabine in TCC cells. Fig. 1 shows that up to $500 \mathrm{nM}$ of gemcitabine did not exert a significant cytotoxic effect on the viability of BFTC905 cells after 48-h treatment $(\mathrm{n}=5)$. Even after $1 \mu \mathrm{M}$ gemcitabine treatment, a moderate but significant reduction of $29.5 \%$ cell viability of BFTC 905 cells was found ( $\mathrm{p}<0.001$ vs. vehicle control). BFTC909 cells were also relatively resistant to gemcitabine, but the viability difference between BFTC905 and BFTC909 cells still reached statistical significance (two-way ANOVA, $\mathrm{p}=0.03$ ). The viability of T24 and TSGH8301 cells was significantly lower than BFTC905 cells after YM155 treatment (two-way ANOVA, both $p<0.001$ ). Thus, among these 4 cell lines examined, BFTC905 was chosen as a model to further investigate the new therapy for gemcitabine-resistant bladder cancer.

Cytotoxicity of YM155 in TCC and primary human urothelial cells. After 48-h treatment, YM155 elicited potent cytotoxicity in a concentration-dependent manner in TCC cells $(n=3$; Fig. 2A). The $\mathrm{IC}_{50}$ s for $4 \mathrm{TCC}$ cell lines were all within $20 \mathrm{nM}$. No significant difference of YM155-induced cytotoxicity between BFTC905 and BFTC909 cells was noted (two-way ANOVA, $p=0.28)$, but YM155 significantly exhibited more potent cytotoxic effects on T24 and TSGH8301 than BFTC905 cells (two-way ANOVA, p<0.001). Within 20-50 nM YM155, the viability between BFTC 905 and T24 or TSGH8301 reached statistically significant difference. The viability of HUCs was not altered after YM155 treatment. Expression of survivin was not detected in HUCs, and the expression of survivin in BFTC905 cells served as a positive control (Fig. 2B).

Cytotoxicity of YM155 plus cisplatin and/or gemcitabine in BFTC905 cells. Next, we evaluated if synergistic or additive cytotoxic effects may be observed when gemcitabine-resistant BFTC905 cells are treated with YM155 plus cisplatin and/or gemcitabine for $48 \mathrm{~h}(\mathrm{n}=3$; Fig. 3). Cisplatin at $2.5 \mu \mathrm{M}$ plus gemcitabine at $100 \mathrm{nM}$ decreased the viability of BFTC 905 cells 
A

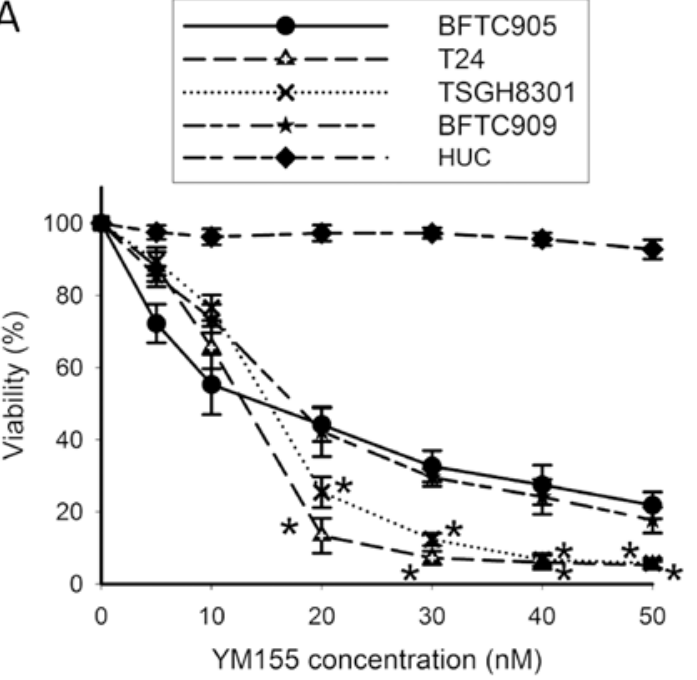

B

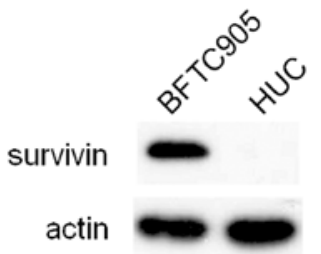

Figure 2. Cytotoxicity of YM155 in TCC cells. (A) The viability of BFTC905, T24, TSGH8301 and BFTC909 cells was measured by MTT assay $48 \mathrm{~h}$ after treatment with YM155. The viability ratio was the ratio of the OD value from the drug-treated sample divided by that of the control sample. ${ }^{*} \mathrm{p}<0.05$ between the difference in viability of TCC cells after YM155 treatment and control. (B) Western blotting of survivin expression in BFTC905 and human urothelial cells (HUCs). Expression of $\alpha$-tubulin was used as an internal standard. TCC, transitional cell carcinoma.

by $13.1 \%(86.9 \pm 2.4 \%$ of viability when compared to control; $\mathrm{p}=0.005)$. When the corresponding concentrations of cisplatin and/or gemcitabine were combined with $20 \mathrm{nM}$ YM155, the cytotoxicities of these combinations were mainly attributed to YM155 (Fig. 3) as $20 \mathrm{nM}$ YM155 alone caused $45 \%$ reduction in the viability of BFTC905 (Fig. 2). Compared to control, any YM155 based regimen showed very potent cytotoxicity on BFTC905 cells ( $\mathrm{p}<0.001)$. No synergistic or additive cytotoxicities in BFTC905 cells were observed when YM155 was combined with cisplatin and/or gemcitabine. YM155 alone provided marked efficacy in treating gemcitabine-resistant TCC cells in vitro.

YM155 inhibits the proliferation of TCC cells. Daily treatment with $20 \mathrm{nM}$ YM155 for 2 days significantly inhibited the proliferation of all TCC cell lines (Fig. 4, n=3, all p<0.001). T2 4 cells were the fastest growing (from $1 \times 10^{5}$ to $9.4 \times 10^{5}$ ), and the most sensitive to inhibition by YM155 (from $9.4 \times 10^{5}$ to $3.8 \times 10^{4}$ ). The slowest growth among the 4 cell lines was TSGH8301 (from $1 \times 10^{5}$ to $4.1 \times 10^{5}$ ) but also the least suppression of proliferation by YM155 (from $4.1 \times 10^{5}$ to $1.3 \times 10^{5}$ ). The proliferation of gemcitabine-resistant BFTC905 and BFTC909 cells were also clearly inhibited (from $7.4 \times 10^{5}$ to $7.7 \times 10^{4}$ and from $6.8 \times 10^{5}$ to $9.3 \times 10^{4}$, respectively). The proliferation of HUCs was not affected following YM155 treatment.

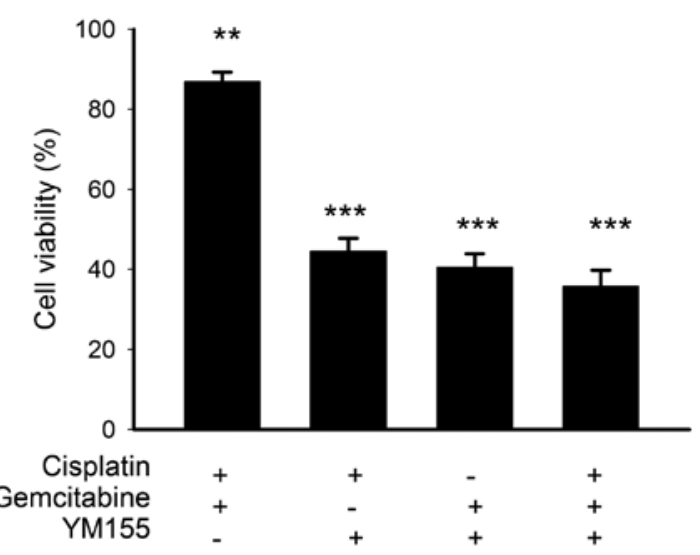

Figure 3. Cytotoxicity of cisplatin and/or gemcitabine plus YM155 in TCC cells. The viability of BFTC905 cells was measured by MTT assay $48 \mathrm{~h}$ after treatment with cisplatin and/or gemcitabine plus YM155. The viability ratio was the ratio of the OD value from the drug-treated sample divided by that of the control sample. ${ }^{* *} \mathrm{p}<0.01$ and ${ }^{* * *} \mathrm{p}<0.005$, between the difference in viability of BFTC905 cells after treatments and control. TCC, transitional cell carcinoma.

YM155 elicits quiescence in BFTC905 cells after YM155 treatment. After 48-h treatment, a significant accumulation in G0/G1 phase $(68.1 \pm 1.1$ vs. $59.4 \pm 0.3 \%$ after YM155 vs. DMSO treatment; $n=3, p=0.002$ ) was observed (Fig. 5A). The expression of cell cycle related proteins is shown in Fig. $5 B$ and $C$. After 48-h treatment, cyclin A/B/D1, CDK4/6, phospho-CDC2 and phospho-CDK2 were significantly decreased. However, cyclin E, p15INK4B and p21 did not show obvious changes. By contrast, the expression of p2 $7^{\mathrm{kip} 1}$ was increased at $48 \mathrm{~h}$ after treatment. These results indicated that no stasis or arrest was found in all proliferation phases of cell cycle, including G1, S and G2/M. We hypothesized that BFTC905 cells were arrested in the G0 phase after YM155 treatment. As shown in Fig. 5D, the expression of proliferation marker Ki-67 was significantly decreased $48 \mathrm{~h}$ after YM155 treatment, indicating that YM155 induced quiescence in BFTC905 cells.

YM155 induces apoptosis in BFTC905 cells. Low basal levels of DNA fragments bound by anti-histone plus anti-DNA antibodies were detected in the culture medium of BFTC905 cells 24 and $48 \mathrm{~h}$ after $20 \mathrm{nM}$ YM155 treatment. A significant amount of cytoplasmic DNA fragments was detected $24 \mathrm{~h}$ after $20 \mathrm{nM}$ YM155 treatment when compared to control $(n=3, p=0.04$; Fig. 6A). By contrast, a significant amount of cytoplasmic DNA fragments was detected $48 \mathrm{~h}$ following YM155 treatment $(n=3, p=0.002$; Fig. 6B). We also examined the morphology of nuclei 24 and $48 \mathrm{~h}$ after $20 \mathrm{nM}$ YM155 treatment using Hoechst 33342 staining. Significantly more nuclear condensation (yellow arrow) in BFTC905 cells was observed after 48 - $h$ treatment $(n=10, p<0.001$; Fig. 6C). Semi-quantitation of the immunofluorescent intensity by flow cytometry revealed significantly increased expression of DNA double-strain break marker phospho-histone $\mathrm{H}_{2} \mathrm{AX}(\mathrm{n}=3$, $\mathrm{p}=0.02$; Fig. 6D) and cleaved PARP $(n=3, p=0.02$; Fig. 6E) $48 \mathrm{~h}$ after treatment with $20 \mathrm{nM}$ YM155. These results indicated that YM155 induced apoptosis of gemcitabine-resistant TCC cells. 

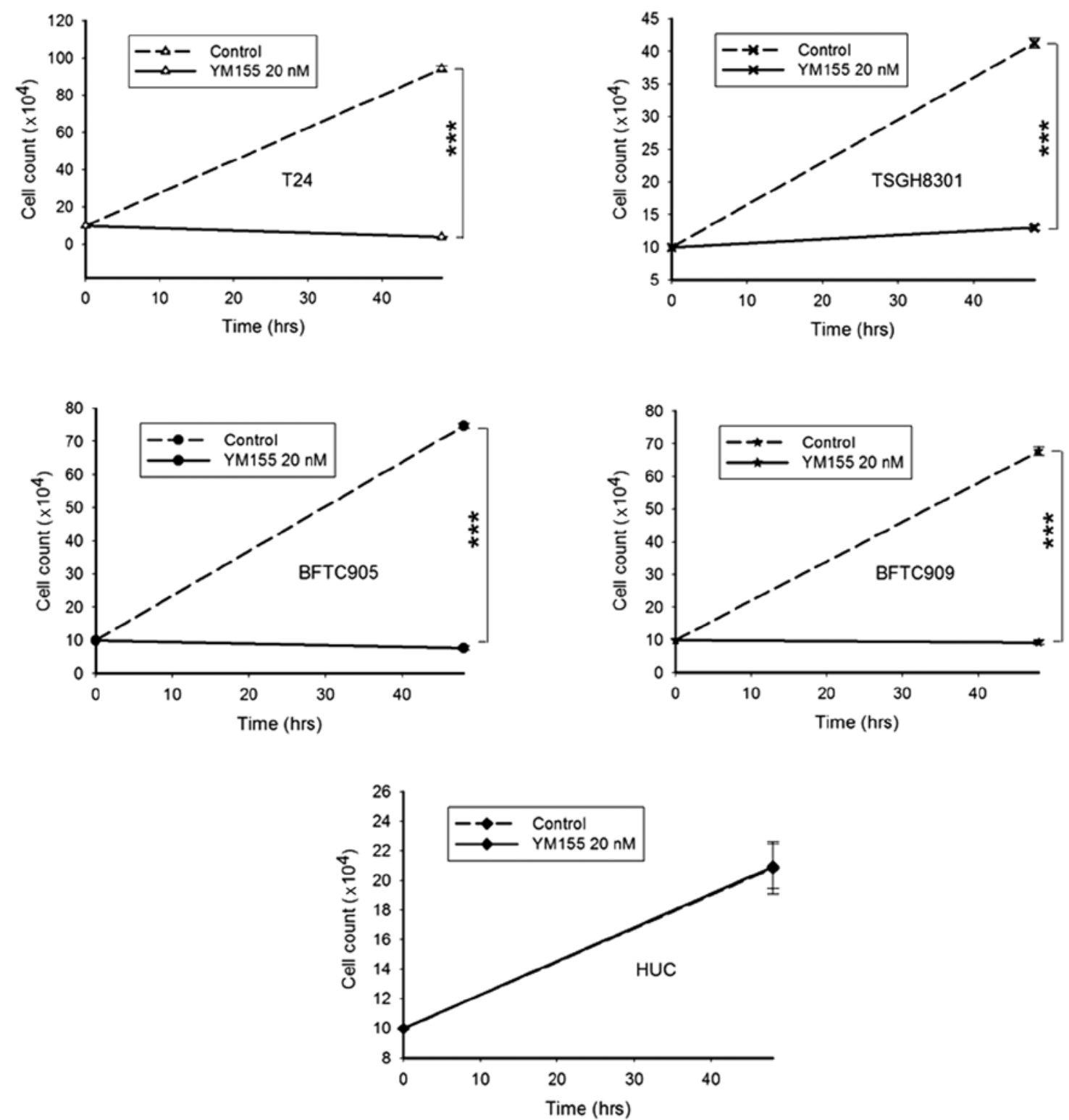

Figure 4. Anti-proliferative effect of YM155 in TCC cells and HUCs. Cell counts of T24, TSGH8301, BFTC905, BFTC909 cells as well as HUCs were calculated $48 \mathrm{~h}$ after $20 \mathrm{nM}$ YM155 treatment once daily. ${ }^{* * * *} \mathrm{p}<0.005$ between the difference in cell counts of TCC cells after YM155 treatment and control. TCC, transitional cell carcinoma; HUCs, human urothelial cells.

YM155 decreases the expression of survivin, securin and bcl-2 in BFTC905 cells. Apart from survivin, securin and bcl-2 are also correlated with the histological or clinical grading of bladder cancer (20-22). Treatment with $1 \mu \mathrm{M}$ gemcitabine for $48 \mathrm{~h}$ did not significantly alter the expression of survivin but it increased the expression of securin and bcl-2 in BFTC905 cells (both $\mathrm{p}=0.03, \mathrm{n}=3$; Fig. 7A). These data may explain the mechanism of gemcitabine resistance in BFTC905 cells. Expressions of survivin, securin and bcl-2 in BFTC905 cells were not significantly altered after 24-h treatment with $20 \mathrm{nM}$ YM155. After 48-h treatment with $20 \mathrm{nM}$ YM155, not only survivin but also securin and bcl-2 were significantly downregulated $(\mathrm{p}=0.006,0.003$ and 0.03 , respectively, $n=3$; Fig. 7B). Translationally controlled tumor protein (TCTP) can prevent apoptosis of cells $(23,24)$. No significant changes of TCTP expression were observed following YM155 treatment.
YM155 induces autophagy in BFTC905 cells. After $20 \mathrm{nM}$ YM155 treatment, autophagic vacuoles detected by Cyto-ID ${ }^{\circledR}$ dye were observed in BFTC905 cells (Fig. 8A). Further flow cytometric analysis showed a significant autophagic flux after YM155 treatment (Fig. 8B). To determine the roles of autophagy in YM155-induced BFTC905 cell death, autophagy inhibitor 3-MA and autophagy inducers sirolimus and everolimus, known as mammalian target of rapamycin (mTOR) inhibitors, were administered additionally with YM155. 3-MA reversed YM155-induced cytotoxicity $(\mathrm{p}=0.04, \mathrm{n}=6)$ while sirolimus and everolimus did not significantly alter YM155-induced cytotoxicity (Fig. 8C). LC3B-II, a marker of autophagy converted from LC3B-I, was also significantly more abundant in BFTC905 cells after $20 \mathrm{nM}$ YM155 treatment ( $\mathrm{p}=0.01$, n=3; Fig. 8D). Concomitantly administered with YM155, 3-MA significantly decreased the expression of LC3B-II ( $p=0.03, n=3)$ while sirolimus and everolimus did not augment its expression (Fig. 8D). 
A
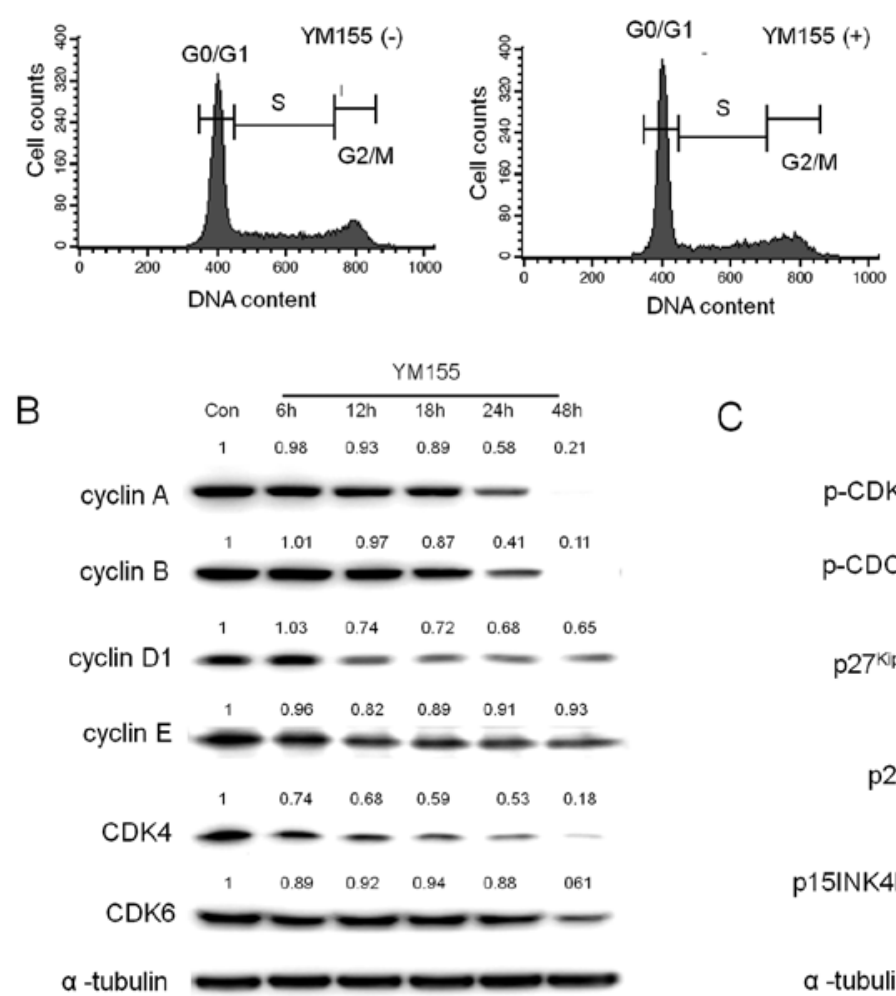

D

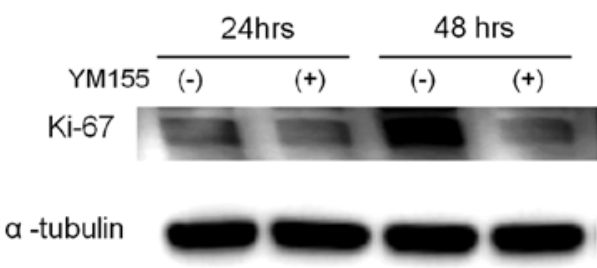

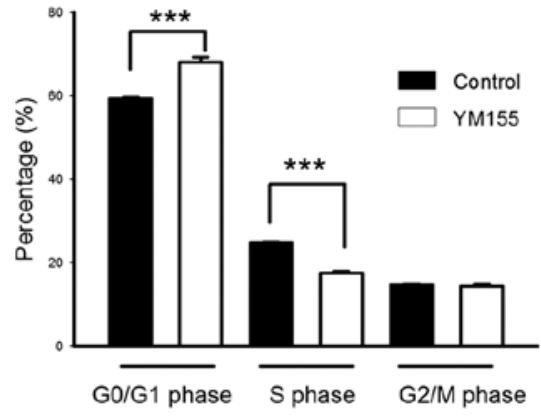

C
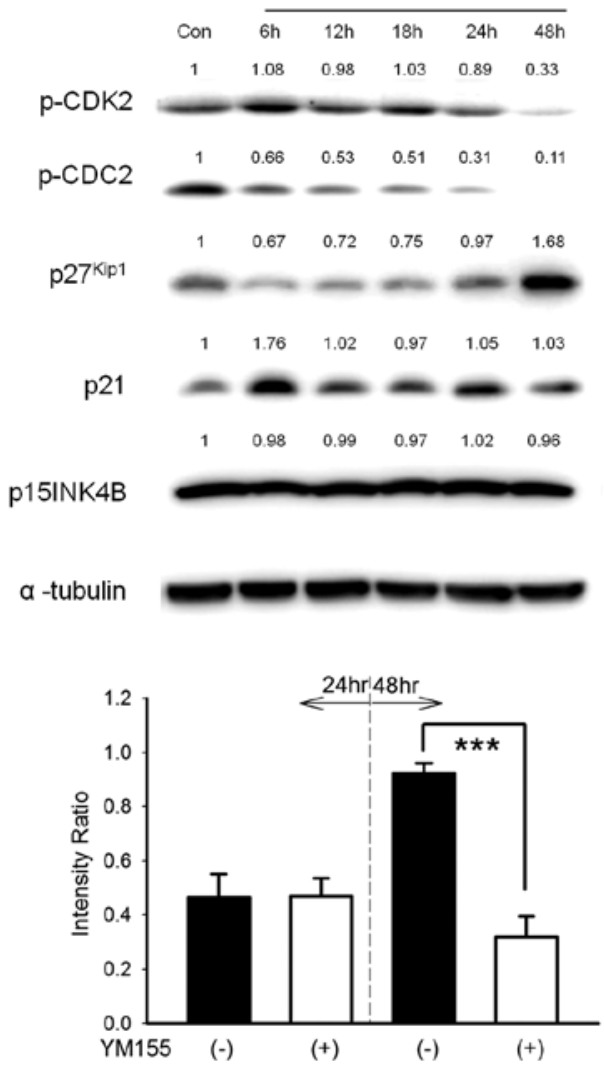

Figure 5. Changes of cell cycle in BFTC905 cells after YM155 treatment. (A) Ratios of cell cycle changes were analyzed by flow cytometry 48 h after YM155 treatment. (B and C) Representative western blotting of survivin expression was examined within $48 \mathrm{~h}$ after treatment with $20 \mathrm{nM}$ YM155. Experiments were repeated 3 times with similar results. The mean intensity ratios shown on the blots were calculated by dividing the intensity of targeted proteins treated with YM155 to control. (D) Representative western blotting of Ki-67 expression was examined 24 and 48 h after treatment with $20 \mathrm{nM}$ YM155. Quantitative comparison of $\mathrm{Ki}-67$ expression is shown in the right hand bar graph. ${ }^{*} \mathrm{p}<0.05$ and ${ }^{* * *} \mathrm{p}<0.005$.

\section{Discussion}

Our results showed that novel survivin inhibitor, YM155, elicited cytotoxicity in TCC cells including gemcitabine-resistant cell line BFTC905. Moreover, no cytotoxic effect was noted in normal human epithelial cells. We demonstrated that treatment with a single agent YM155 already provided marked therapeutic effects through multiple mechanisms. Few phase I/II clinical trials of YM155 for cancer therapies have been reported and it was well tolerated by patients with minimal adverse effects such as fatigue and nausea (25). Thus, YM155 may offer a new hope for refractory bladder cancer patients.

Very low $\mathrm{IC}_{50}$ s of gemcitabine (usually $<100 \mathrm{nM}$ ) have been reported for many TCC cell lines $(26,27)$ except one gemcitabine-resistant TCC cell line UM-UC-3R (8). $\mathrm{IC}_{50} \mathrm{~s}$ of gemcitabine for T24 and TSGH8301 cells in our experiments were $>100 \mathrm{nM}$, and we suppose the variation may result from different cytotoxic assays and protocols. However, the viability of T24 and TSGH8301 cells was indeed much lower than BFTC905 cells after gemcitabine treatment. Gemcitabine upregulated survivin expression in pancreatic cancer MiaPaCa-2 cells, but this phenomenon was not observed in BFTC905 cells (28). Securin, the product of pituitary tumortransforming gene (PTTG) (29), has been reported to mediate gefitinib resistance in cancer cells (30). Bcl-2, an anti-apoptotic protein, has also been reported to mediate cisplatin resistance in urothelial carcinoma cells (31). In the present investigation, upregulation of securin and bcl-2 in BFTC905 cells may be a compensatory mechanism for gemcitabine resistance. We have also reported that only BFTC905 but not T24 and TSGH8301 xenografts successfully grew in SCID mice with less cell loading (32). Collectively, the above observations indicate that BFTC905 is the first choice of TCC cell lines for investigating the new therapy for gemcitabine-resistant bladder cancer. 

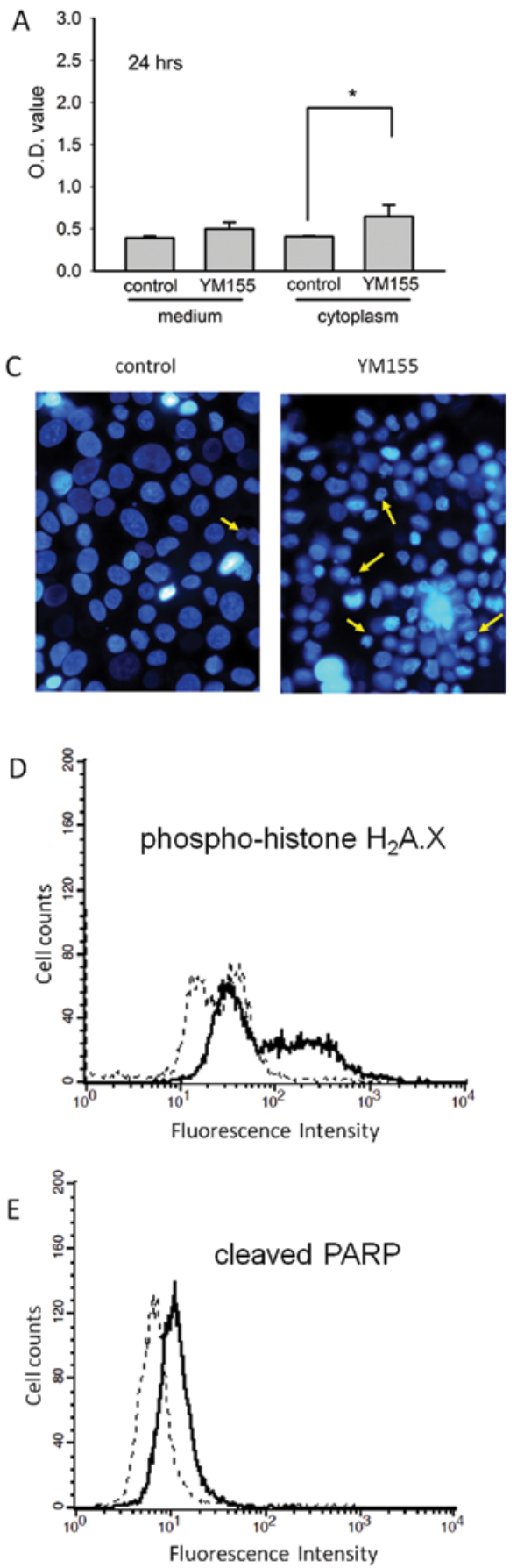
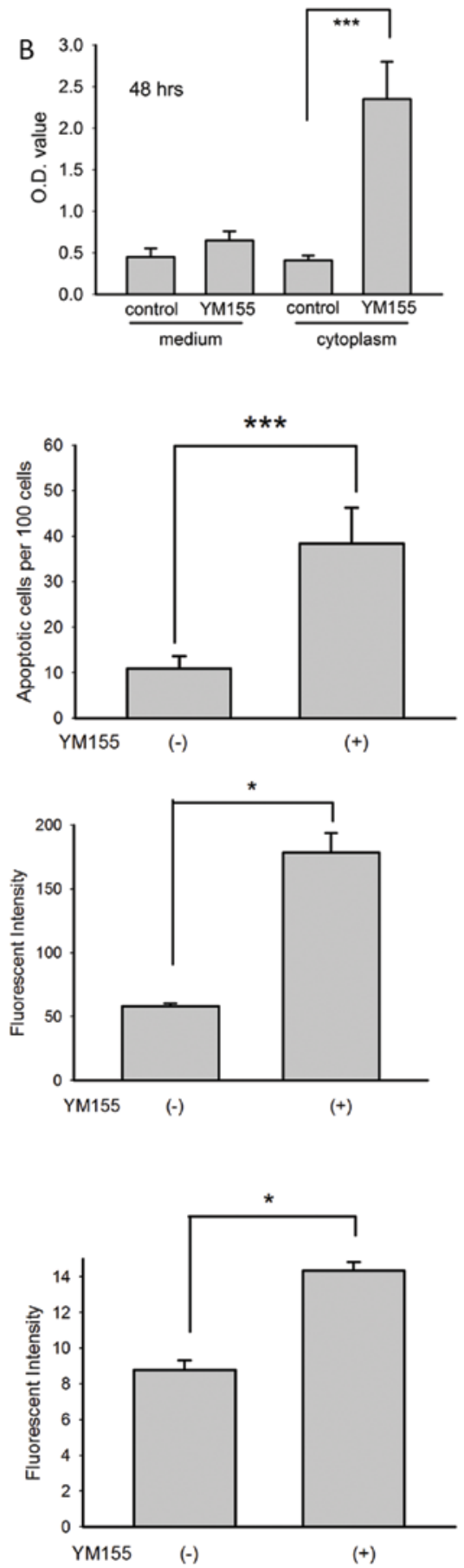

Figure 6. Apoptosis in BFTC905 cells after YM155 treatment. After treatment with $20 \mathrm{nM}$ YM155 for (A) $24 \mathrm{~h}$ and (B) $48 \mathrm{~h}$, the supernatants of both the culture medium and the cytoplasmic fraction of BFTC905 cells were collected. Increased expression of DNA fragments in the medium indicates necrosis due to membrane rupture, while increased expression in the cytoplasm alone indicates apoptosis. (C) Nuclear condensation (yellow arrow) in BFTC905 cells was observed after 48-h treatment with YM155 by Hoechst 33342 staining. Quantitative comparison of apoptotic cells is shown in the right hand bar graph. Immunofluorescent intensity of (D) phospho-histone $\mathrm{H}_{2} \mathrm{AX}$ and (E) cleaved PARP after 48-h YM155 treatment, measured by flow cytometry. Immunofluorescent distribution in solid and dash lines indicates treatment with YM155 and control, respectively. ${ }^{*} \mathrm{p}<0.05$ and ${ }^{* * * *} \mathrm{p}<0.005$.

Our results showed that YM155 caused potent cytotoxicity in TCC cells, including gemcitabine-resistant ones. It may result from survivin similarly expressed in these cell lines (33). However, other different characteristics that exist among BFTC905 and other high grade TCC cell lines should be further investigated. Since survivin is not expressed in HUCs, it is reasonable that YM155 did not elicit cytotoxic effects in HUCs. YM155 acting on bladder cancer cells without affecting normal urothelium would be the best choice for targeted therapy. Cisplatin plus gemcitabine is the standard chemotherapy regimen for bladder cancer, but fair cytotoxic effect was noted in BFTC905 cells. YM155 has been reported to reverse cisplatin resistance in head and neck squamous cell carcinoma cells (34). Thus, we also tested the cytotoxic effects of YM155 combined with cisplatin, gemcitabine, or both. YM155 has been reported to potentiate the therapeutic effects 

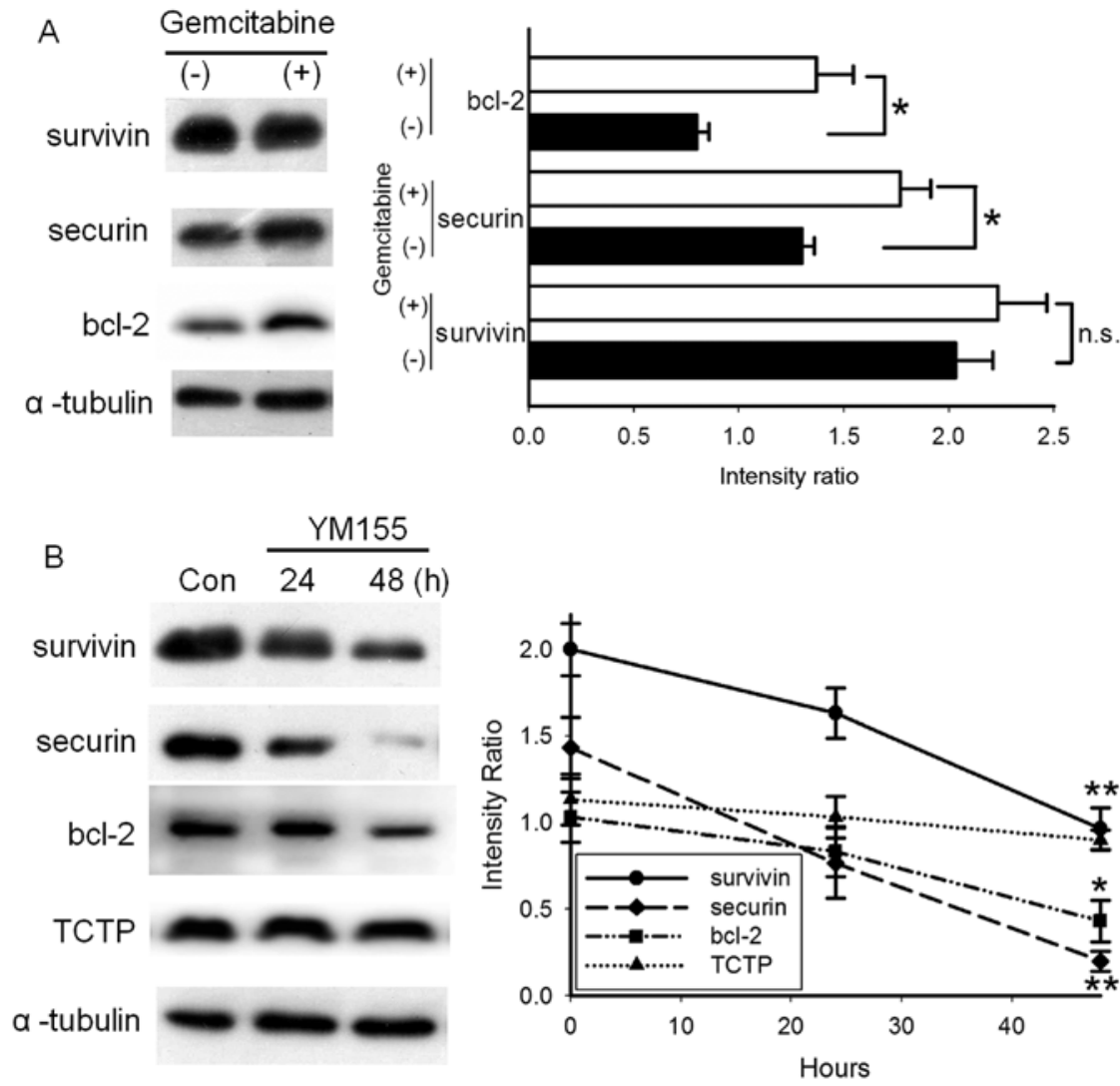

Figure 7. Expression of survivin, securin, bcl-2 and TCTP in BFTC905 cells after gemcitabine or YM155 treatment. (A) Representative western blotting of survivin, securin and bcl-2 expression were examined $48 \mathrm{~h}$ after treatment with $1 \mu \mathrm{M}$ gemcitabine. (B) Representative western blotting of survivin, securin, bcl-2 and TCTP expression were examined 24 and $48 \mathrm{~h}$ after treatment with $20 \mathrm{nM}$ YM155. Quantitative comparison of the expression is shown in the right hand figures. ${ }^{*} \mathrm{p}<0.05$ and ${ }^{* *} \mathrm{p}<0.01$. TCTP, translationally controlled tumor protein.

of many chemotherapy or targeted-therapy agents (13,14,35-39). However, our results demonstrated that YM155 alone exerted potent cytotoxicity in BFTC905 cells, and addition of cisplatin and/or gemcitabine to YM155 did not elicit synergistic or additive effects. The present study also confirmed that survivin plays a key role in the survival of TCC cells. YM155 may be a suitable option for refractory bladder cancer therapy.

Survivin, a member of the inhibitor of apoptosis (IAP) family, is abundantly expressed in the $\mathrm{G} 2 / \mathrm{M}$ phase of the cell cycle to regulate mitosis (40). Downregulation of survivin by siRNA inhibited bladder cancer growth with $\mathrm{G} 2 / \mathrm{M}$ phase arrest (41). However, survivin inhibitor YM155 caused variable cell cycle arrest and even a lack of changes in cell cycles were found in previous reports. In malignant peripheral nerve sheath tumor cells, YM155 caused G2/M cell cycle arrest (42). Cheng et al demonstrated a slight $\mathrm{S}$ phase stasis in PC3 prostate cancer cells after 24- and 48-h YM155 treatment (43). Since cell cycle analysis using flow cytometry only represents the proportional changes in each phase after treatment, we further examined the specific cell cycle related proteins. The roles of cyclins, cyclin dependent kinases and related molecules in cancer cell cycles have been well established $(44,45)$. Downregulation of $\mathrm{G} 2 / \mathrm{M}$ phase marker cyclin B and phospho-CDC2 in the present study indicated no G/M arrest. Downregulation of cyclin $\mathrm{A}$, which is expressed in $\mathrm{S}$ phase, indicated no $\mathrm{S}$ phase arrest. CDK2 is expressed in late $\mathrm{G} 1$ and $\mathrm{S}$ phase, therefore a decrease in CDK2 expression indicated no cell cycle stasis in late G1 and S phase. Cyclin D1 and CDK4/6 are expressed in G1 phase before R point, while cyclin $\mathrm{E}$ is expressed in late G1 phase after R point. Expression of these markers was all decreased after YM155 treatment; thus, YM155 did not induce G1 phase arrest. Due to the increased ratio of G0/G1 phase without G1 arrest, YM155 induced G0 phase stasis, known as quiescence, was substantiated. Our results are consistent with those of Arora et al who demonstrated the early inhibition of DNA synthesis in Merkel cell carcinoma by YM155 (46). After 48-h YM155 treatment, the increased expression of $\mathrm{p} 27^{\mathrm{kip} 1}$, a potent inhibitor of cyclin-CDK complex formation, supported this idea. $\mathrm{Ki}-67$ protein is present in the nucleus during all active phases of the cell cycle (G1, S, G2 and mitotic phase) but absent from quiescent cells (G0), so Ki-67 is the most referenced marker for cellular proliferation $(47,48)$. In our results, expression of Ki-67 after 48-h YM155 treatment was much lower than control. Thus, we found the novel effect of YM155 in promoting quiescence in gemcitabine-resistant TCC cells.

Survivin plays important roles in anti-apoptosis, so any method to decrease the expression of survivin in bladder cancer cells should elicit apoptosis $(49,50)$. In this regard, survivin inhibitor YM155 promoted apoptosis of cancer cells $(19,51)$, and we also observed that YM155 induced apoptosis in TCC cells with enhanced expression of cleaved PARP and phospho-histone $\gamma$-H2AX. PARP is involved in the repair of single-strand DNA nicks, but cleaved PARP facilitates cellular disassembly and promotes ongoing apoptosis (52). Enhanced 
A

YM155 (-)

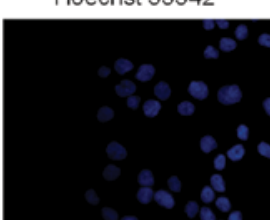

YM155 (+)

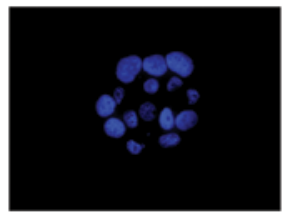

B

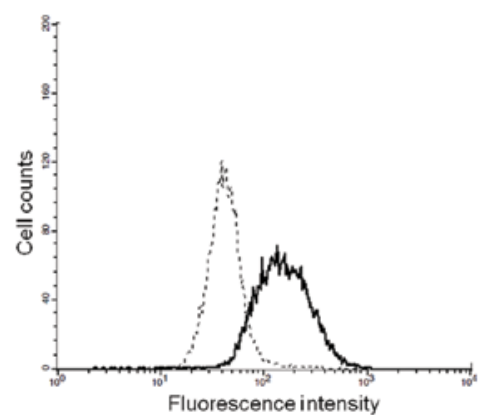

Cyto-ID
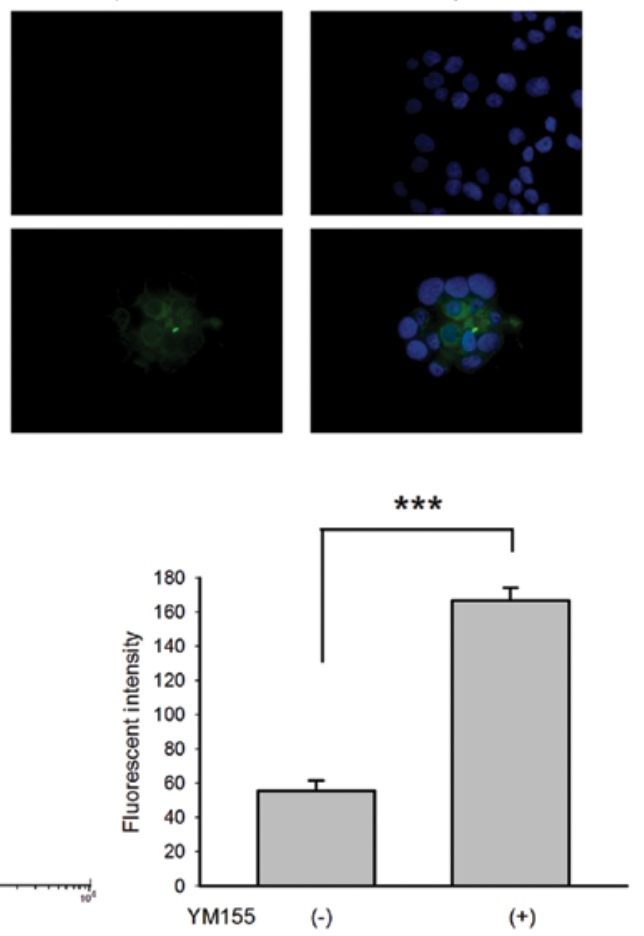

C

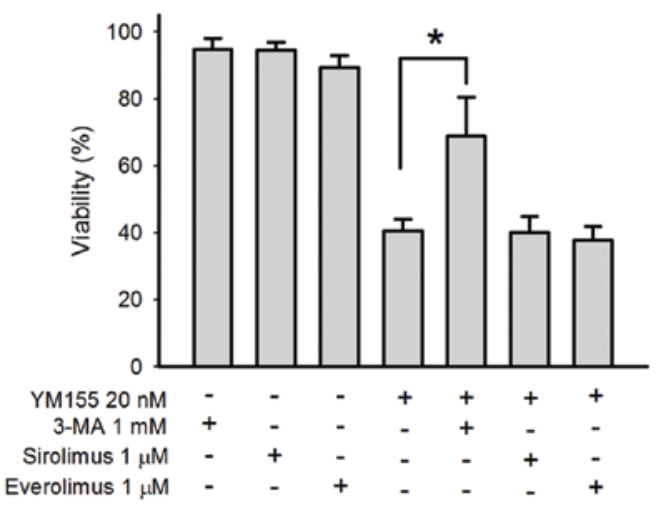

merge
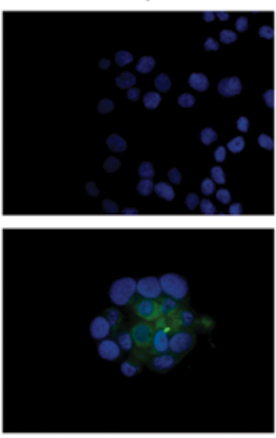

(+)$$
\text { . }
$$

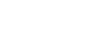

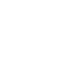


YM155 treatment, for instance, an increase in non-small cell lung cancer Calu6 cells but no changes in H460 cells were reported (35). Similarly, expression of bcl-2 in A375 melanoma cells was not altered after YM155 treatment (38), but marked inhibition of bcl-2 expression by survivin siRNA in human renal clear cell carcinoma 786-O cells, similar to our results, was observed (56). It may result from the characteristics of individual cell lines. Securin was found as an inhibitor of premature sister chromatid separation in nuclei (57). In our previous report, securin was overexpressed in human TCC specimens when compared to normal urothelium (22). Chao and Liu also demonstrated that survivin siRNA significantly induced apoptosis and decreased the levels of securin in A549 cells (58). We have also reported YM155-induced apoptosis with securin suppression in glioblastoma multiforme cells (32). Overexpression of TCTP was observed in many malignancies (12), but whether TCTP is expressed in bladder cancer has not be determined. Our results demonstrated the first observation of TCTP expression in TCC cells. Expression of TCTP in the rat urothelium has been reported (59). No changes in TCTP expression after YM155 treatment suggest that the function of urothelium may not be altered to induce adverse effects. The detailed signaling among survivin, securin and bcl-2 in TCC should be further investigated.

Survivin is also involved in autophagy. Survivin controls autophagosome formation and protects cancer cells from autophagic death, known as type II cell death, by inhibiting the expression of LC3II (60). In prostate cancer cells, YM155 induced autophagy and autophagy inhibitor 3-MA attenuated YM155-induced apoptosis (12). In that study, autophagy induced by YM155 played a pro-apoptotic role. However, autophagy is a double-edged sword (61). Autophagy is also important for survival in tumor cells by overcoming hypoxia and a shortage of nutrients. Therefore, we evaluated the role of YM155-induced autophagy in BFTC905 cells. As expected, 3-MA diminished YM155-induced cytotoxicity by decreasing the expression of LC3B-II. Sirolimus and everolimus, however, did not elicit additive cytotoxic effects with YM155 in BFTC905 cells. Compared to treatment with YM155 alone, expression of LC3B-II was not increased after YM155 plus mTOR inhibitors, indicating that YM155 per se is a strong activator of LC3B-II. YM155-induced autophagy promoted the death of BFTC 905 cells.

In conclusion, low concentrations of YM155 induced quiescence, apoptosis, and autophagy in gemcitabine-resistant human TCC cells without causing cytotoxicity in normal human urothelium. YM155 may become a new candidate for advanced or metastatic bladder cancer therapy.

\section{Acknowledgements}

The present study was supported by a grant-in-aid from Tzu Chi General Hospital, Hualien 970, Taiwan.

\section{References}

1. Tavora F and Epstein JI: Bladder cancer, pathological classification and staging. BJU Int 102: 1216-1220, 2008.

2. Murta-Nascimento C, Schmitz-Dräger, Zeegers MP, et al: Epidemiology of urinary bladder cancer: from tumor development to patient's death. World J Urol 25: 285-295, 2007.
3. George L, Bladou F, Bardou VJ, et al: Clinical outcome in patients with locally advanced bladder carcinoma treated with conservative multimodality therapy. Urology 64: 488-493, 2004.

4. von der Maase H, Hansen SW, Roberts JT, et al: Gemcitabine and cisplatin versus methotrexate, vinblastine, doxorubicin, and cisplatin in advanced or metastatic bladder cancer: results of a large, randomized, multinational, multicenter, phase III study. J Clin Oncol 18: 3068-3077, 2000.

5. Sternberg CN: Gemcitabine in bladder cancer. Semin Oncol 27: 31-39, 2000.

6. Chaudhary UB, Verma N, Keane T and Gudena V: A phase II study of gemcitabine and irinotecan in patients with locally advanced or metastatic bladder cancer. Am J Clin Oncol: Dec 13, 2012 (Epub ahead of print)

7. Culine S, Fléchon A, Guillot A, et al: Gemcitabine or gemcitabine plus oxaliplatin in the first-line treatment of patients with advanced transitional cell carcinoma of the urothelium unfit for cisplatin-based chemotherapy: a randomized phase 2 study of the French Genitourinary Tumor Group (GETUG V01). Eur Urol 60: 1251-1257, 2011.

8. Muramaki M, So A, Hayashi N, et al: Chemosensitization of gemcitabine-resistant human bladder cancer cell line both in vitro and in vivo using antisense oligonucleotide targeting the anti-apoptotic gene, clusterin. BJU Int 103: 384-390, 2009.

9. Margulis V, Lotan Y and Shariat SF: Survivin: a promising biomarker for detection and prognosis of bladder cancer. World J Urol 26: 59-65, 2008.

10. Nakahara T, Takeuchi M, Kinoyama I, et al: YM155, a novel small-molecule survivin suppressant, induces regression of established human hormone-refractory prostate tumor xenografts. Cancer Res 67: 8014-8021, 2007.

11. Nakamura N, Yamauchi T, Hiramoto M, et al: Interleukin enhancer-binding factor 3/NF110 is a target of YM155, a suppressant of survivin. Mol Cell Proteomics 11: M111. 013243, 2012.

12. Wang Q, Chen Z, Diao X and Huang S: Induction of autophagydependent apoptosis by the survivin suppressant YM155 in prostate cancer cells. Cancer Lett 302: 29-36, 2011.

13. Nakahara T, Yamanaka K, Hatakeyama S, et al: YM155, a novel survivin suppressant, enhances taxane-induced apoptosis and tumor regression in a human Calu 6 lung cancer xenograft model. Anticancer Drugs 22: 454-462, 2011.

14. Iwasa T, Okamoto I, Suzuki M, et al: Radiosensitizing effect of YM155, a novel small-molecule survivin suppressant, in non-small cell lung cancer cell lines. Clin Cancer Res 14: 6496-6504, 2008.

15. Tzeng CC, Liu HS, Li C, et al: Characterization of two urothelium cancer cell lines derived from a blackfoot disease endemic area in Taiwan. Anticancer Res 16: 1797-1804, 1996.

16. Huang YT, Lai PC, Wu CC, et al: BDNF mediated TrkB activation is a survival signal for transitional cell carcinoma cells. Int $J$ Oncol 36: 1469-1476, 2010.

17. Lai PC, Yang YC, Cheng CC, Chiu TH and Huang YT: Brain-derived neurotrophic factor plus vascular endothelial growth factor additively promotes early growth of the transitional cell carcinoma cell line BFTC905 in vitro and in vivo. Tzu Chi Med J 25: 155-160, 2013.

18. Chao JI, Su WC and Liu HF: Baicalein induces cancer cell death and proliferation retardation by the inhibition of CDC2 kinase and survivin associated with opposite role of p38 mitogen-activated protein kinase and AKT. Mol Cancer Ther 6: 3039-3048, 2007.

19. Lai PC, Chen SH, Yang SH, Cheng CC, Chiu TH and Huang YT: Novel survivin inhibitor YM155 elicits cytotoxicity in glioblastoma cell lines with normal or deficiency DNA-dependent protein kinase activity. Pediatr Neonatol 53: 199-204, 2012.

20. Liukkonen TJ, Lipponen PK, Helle M and Jauhiainen KE: Immunoreactivity of bcl-2, p53 and EGFr is associated with tumor stage, grade and cell proliferation in superficial bladder cancer. Finnbladder III Group. Urol Res 25: 1-7, 1997.

21. Gazzaniga P, Gradilone A, Silvestri I, et al: Variable levels of bcl-2, bcl-X and bax mRNA in bladder cancer progression. Oncol Rep 5: 901-904, 1998.

22. Lai PC, Fang TC, Chiu TH and Huang YT: Overexpression of securin in human transitional cell carcinoma specimens. Tzu Chi Med J 22: 171-176, 2010.

23. Bommer UA and Thiele BJ: The translationally controlled tumour protein (TCTP). Int J Biochem Cell Biol 36: 379-385, 2004.

24. Koziol MJ and Gurdon JB: TCTP in development and cancer. Biochem Res Int 2012: 105203, 2012. 
25. Lewis KD, Samlowski W, Ward J, et al.: A multi-center phase II evaluation of the small molecule survivin suppressor YM155 in patients with unresectable stage III or IV melanoma. Invest New Drugs 29: 161-166, 2011.

26. Yoon CY, Lee JS, Kim BS, et al: Sunitinib malate synergistically potentiates anti-tumor effect of gemcitabine in human bladder cancer cells. Korean J Urol 52: 55-63, 2011.

27. Moibi JA, Mak AL, Sun B and Moore RB: Urothelial cancer cell response to combination therapy of gemcitabine and TRAIL. Int J Oncol 39: 61-71, 2011.

28. Yoon DH, Shin JS, Jin DH, et al: The survivin suppressant YM155 potentiates chemosensitivity to gemcitabine in the human pancreatic cancer cell line MiaPaCa-2. Anticancer Res 32: 1681-1688, 2012.

29. Zou H, McGarry TJ, Bernal T and Kirschner MW: Identification of a vertebrate sister-chromatid separation inhibitor involved in transformation and tumorigenesis. Science 285: 418-422, 1999.

30. Yu SY, Liu HF, Wang SP, Chang CC, Tsai CM and Chao JI: Evidence of securin-mediated resistance to gefitinib-induced apoptosis in human cancer cells. Chem Biol Interact 203: 412-422, 2013.

31. Yu HM and Wang TC: Mechanism of cisplatin resistance in human urothelial carcinoma cells. Food Chem Toxicol 50: 1226-1237, 2012

32. Lai PC, Chiu TH and Huang YT: Overexpression of BDNF and TrkB in human bladder cancer specimens. Oncol Rep 24 $1265-1270,2010$

33. Huang YT, Lai PC, Wu CC, Cheng CC and Chiu TH: TrkB antibody elicits cytotoxicity and suppresses migration/invasion of transitional cell carcinoma cells. Int J Oncol 37: 943-949, 2010.

34. Kumar B, Yadav A, Lang JC, et al: YM155 reverses cisplatin resistance in head and neck cancer by decreasing cytoplasmic survivin levels. Mol Cancer Ther 11: 1988-1998, 2012

35. Iwasa T, Okamoto I, Takezawa K, et al: Marked anti-tumour activity of the combination of YM155, a novel survivin suppressant, and platinum-based drugs. Br J Cancer 103: 36-42, 2010.

36. Kita A, Mitsuoka K, Kaneko N, et al: Sepantronium bromide (YM155) enhances response of human B-cell non-Hodgkin lymphoma to rituximab. J Pharmacol Exp Ther 343: 178-183, 2012.

37. Kaneko N, Kita A, Yamanaka K and Mori M: Combination of YM155, a survivin suppressant with a STAT3 inhibitor: a new strategy to treat diffuse large B-cell lymphoma. Leuk Res 37: 1156-1161,2013.

38. Yamanaka K, Nakahara T, Yamauchi T, et al: Antitumor activity of YM155, a selective small-molecule survivin suppressant, alone and in combination with docetaxel in human malignant melanoma models. Clin Cancer Res 17: 5423-5431, 2011.

39. Chen J, Pise-Masison CA, Shih JH, et al: Markedly additive antitumor activity with the combination of a selective survivin suppressant YM155 and alemtuzumab in adult T-cell leukemia. Blood 121: 2029-2037, 2013.

40. Li F, Ambrosini G, Chu EY, et al: Control of apoptosis and mitotic spindle checkpoint by survivin. Nature 396: 580-584, 1998.

41. Ning S, Fuessel S, Kotzsch M, et al: siRNA-mediated downregulation of survivin inhibits bladder cancer cell growth. Int J Oncol 25: 1065-1071, 2004

42. Ghadimi MP, Young ED, Belousov R, et al: Survivin is a viable target for the treatment of malignant peripheral nerve sheath tumors. Clin Cancer Res 18: 2545-2557, 2012.
43. Cheng Q, Ling X, Haller A, et al: Suppression of survivin promoter activity by YM155 involves disruption of Sp1-DNA interaction in the survivin core promoter. Int $\mathrm{J}$ Biochem Mol Biol 3: 179-197, 2012.

44. Sherr CJ: Cancer cell cycles. Science 274: 1672-1677, 1996.

45. Hunter T and Pines J: Cyclins and cancer. II: cyclin D and CDK inhibitors come of age. Cell 79: 573-582, 1994.

46. Arora R, Shuda M, Guastafierro A, et al: Survivin is a therapeutic target in Merkel cell carcinoma. Sci Transl Med 4: 133ra56, 2012.

47. Scholzen T and Gerdes J: The Ki-67 protein: from the known and the unknown. J Cell Physiol 182: 311-322, 2000.

48. Gerdes J, Li L, Schlueter C, et al: Immunobiochemical and molecular biologic characterization of the cell proliferationassociated nuclear antigen that is defined by monoclonal antibody Ki-67. Am J Pathol 138: 867-873, 1991.

49. Gou X, Yang HA, He WY, Xioa MC and Wang M: Gene silence-induced downregulation of survivin inhibits bladder cancer cells. Oncol Res 19: 535-541, 2011

50. Ku JH, Seo SY, Kwak C and Kim HH: Cytotoxicity and apoptosis by survivin small interfering RNA in bladder cancer cells. BJU Int 106: 1812-1816, 2010.

51. Tao YF, Lu J, Du XJ, et al: Survivin selective inhibitor YM155 induce apoptosis in SK-NEP-1 Wilms tumor cells. BMC Cancer 12: 619, 2012.

52. Oliver FJ, de la Rubia G, Rolli V, Ruiz-Ruiz MC, de Murcia G and Murcia JM: Importance of poly(ADP-ribose) polymerase and its cleavage in apoptosis. Lesson from an uncleavable mutant. J Biol Chem 273: 33533-33539, 1998

53. Yamanaka K, Nakata M, Kaneko N, et al: YM155, a selective survivin suppressant, inhibits tumor spread and prolongs survival in a spontaneous metastatic model of human triple negative breast cancer. Int J Oncol 39: 569-575, 2011.

54. Kuo LJ and Yang LX: $\gamma-\mathrm{H} 2 \mathrm{AX}$ - a novel biomarker for DNA double-strand breaks. In Vivo 22: 305-309, 2008.

55. Chowdhury D, Keogh MC, Ishii H, Peterson CL, Buratowski S and Lieberman J: $\gamma$-H2AX dephosphorylation by protein phosphatase 2A facilitates DNA double-strand break repair. Mol Cell 20: 801-809, 2005.

56. Zhang Y, Chen ZD, Du CJ, Xu G and Luo W: siRNA targeting survivin inhibits growth and induces apoptosis in human renal clear cell carcinoma 786-O cells. Pathol Res Pract 205: 823-827, 2009.

57. Salehi F, Kovacs K, Scheithauer BW, Lloyd RV and Cusimano M: Pituitary tumor-transforming gene in endocrine and other neoplasms: a review and update. Endocr Relat Cancer 15: 721-743, 2008.

58. Chao JI and Liu HF: The blockage of survivin and securin expression increases the cytochalasin B-induced cell death and growth inhibition in human cancer cells. Mol Pharmacol 69: 154-164, 2006.

59. Sheverdin V, Bae SY, Shin DH and Lee K: Expression and localization of translationally controlled tumor protein in rat urinary organs. Microsc Res Tech 75: 1576-1581, 2012.

60. Roca H, Varsos ZS, Mizutani K and Pienta KJ: CCL2, survivin and autophagy: new links with implications in human cancer. Autophagy 4: 969-971, 2008

61. Kreuzaler P and Watson CJ: Killing a cancer: what are the alternatives? Nat Rev Cancer 12: 411-424, 2012. 